\title{
Correction to: Platelet-rich plasma as a potential therapeutic approach against lead nitrate- and/or gamma radiation-induced hepatotoxicity
}

\author{
Salma M. Abdel Fattah ${ }^{1} \cdot$ Mostafa Saif-Elnasr $^{2} \cdot$ Ahmed F. Soliman $^{3}$
}

Published online: 5 November 2018

(C) Springer-Verlag GmbH Germany, part of Springer Nature 2018

\section{Correction to: Environmental Science and Pollution Research} https://doi.org/10.1007/s11356-018-3366-3

The original publication of this paper contains adjustment errors.

The images for figures 1 and 2 were adjusted and shown in the original paper.

The original article has been corrected.

The online version of the original article can be found at https://doi.org/ 10.1007/s11356-018-3366-3

Salma M. Abdel Fattah

salmaelbanna@gmail.com

1 Drug Radiation Research Department, National Center for Radiation Research and Technology, Egyptian Atomic Energy Authority, Cairo, Egypt

2 Health Radiation Research Department, National Center for Radiation Research and Technology, Egyptian Atomic Energy Authority, Cairo, Egypt

3 Biochemistry Department, Faculty of Science, Ain Shams University, Cairo, Egypt 